\title{
Radiation Effect on Unsteady Free Convection and Mass Transfer Flow Between Two Vertical Parallel Plates with Newtonian Heating
}

\author{
Fasihah Zulkiflee* and Ahmad Qushairi Mohamadand Sharidan Shafie \\ Department of Mathematical Sciences, Faculty of Science, Universiti Teknologi Malaysia, 81310 UTM Johor
}

Bahru, Johor, Malaysia

\begin{abstract}
The purpose of this study is to investigate analytically free convection flow between two vertical parallel plates with Newtonian heating and mass diffusion. Radiation also taken into consideration. Appropriate non-dimensional variables are used to transform the dimensional governing equations. The velocity, temperature and concentration profiles are solved analytically using Laplace Transform technique. The corresponding expressions for skin friction and Nusselt number are also calculated. The graphical results are displayed to illustrate the influence of Newtonian heating parameter, thermal radiation parameter, Prandtl number and Schmidt number. The effect of Newtonian heating parameter is observed whereby it increases the Nusselt number but decreases the skin friction. Results also shown that, Nusselt number decreases when radiation increases meanwhile skin friction decreases when radiation parameter increases.
\end{abstract}

Keywords: Free convection flow, vertical parallel plates, Newtonian heating, Radiation, mass transfer.

\section{INTRODUCTION}

The importance of free convection in vertical channel has been studied widely as it has connection with many engineering applications such as cooling of electric equipment, cooling of nuclear reactors, chemical devices and others. Free convection in vertical channel has been studied with different physical effects such as radiation, heat generation/absorption, chemical reaction, magnetohydrodynamics and others. Previous research has investigated combined effects of chemical reactions and heat generation and absorption through porous medium with constant temperature (Rajput \& Sahu,2011a). While MHD effect with symmetric heating was investigated by Basant et. al., (2011).

Generally, the problems of free convection flows in parallel plate are usually modelled under the assumptions of constant surface temperature, ramped wall temperature, or constant surface heat flux (Rajput \& Sahu, 2011b; Singh et. al., 1996; Narahari, 2012; Narahari, 2008; Paul et. al., 1996). However, in many practical situations where the heat transfer from the surface is taken to be proportional to the local surface temperature, the above assumptions fail to work. Such types of flows are termed as conjugate convective flows, and the proportionally condition of the heat transfer to the local surface temperature is termed as Newtonian heating. Lesnic et. al., (2004) investigated free convection boundary layer flow above a nearly horizontal surface in a porous medium with Newtonian heating while Shehzad et. al., (2014) investigate threedimensional flow of Jeffrey fluid with Newtonian heating. There are many researchers consider Newtonian heating in their research but not many considered Newtonian heating in between two parallel plate. The literature survey shows that research in Newtonian heating

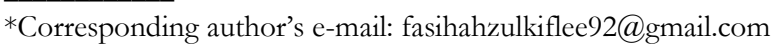


between two parallel plates are very few (Hamza, 2016). The present study is an attempt to investigate the effect of radiation on unsteady flow between two parallel plates with Newtonian heating effect.

\section{MATHEMATICAL ANALYSIS}

Consider an unsteady free convection flow between two parallel plates with presence of radiation. Newtonian heating also considered in this study. The $x^{\prime}$-axis is considered along one of the vertical plates and the $y^{\prime}$-axis is taken normal to the plates. Figure 1 shows the flow configuration and coordinates systems of the flow and the governing equations under the normal Boussinesq's approximation are as follows.

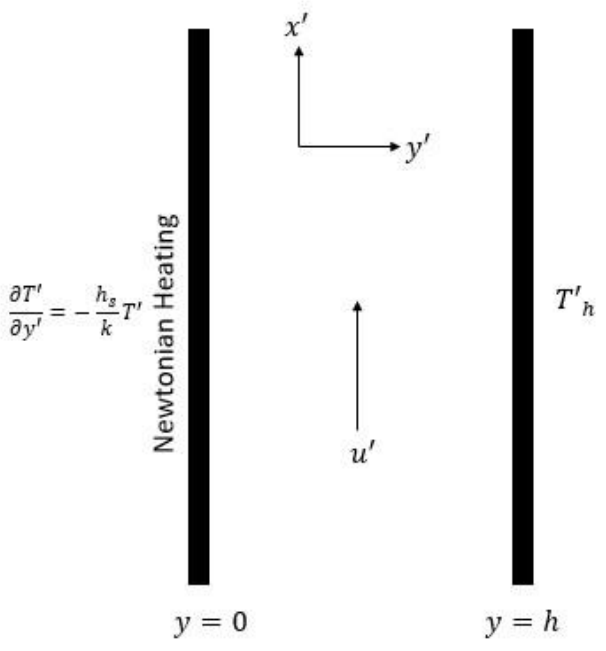

Figure 1. Flow configuration of the problem

$$
\begin{gathered}
\frac{\partial u^{\prime}}{\partial t^{\prime}}=\nu \frac{\partial^{2} u^{\prime}}{\partial y^{\prime 2}}+g \beta\left(T^{\prime}-T_{h}^{\prime}\right)+g \beta^{*}\left(C^{\prime}-C_{h}^{\prime}\right) \\
\rho C_{p} \frac{\partial T^{\prime}}{\partial t^{\prime}}=k \frac{\partial^{2} T^{\prime}}{\partial y^{\prime 2}}-\frac{\partial q_{r}}{\partial y^{\prime}} \\
\frac{\partial C^{\prime}}{\partial t^{\prime}}=D \frac{\partial^{2} C^{\prime}}{\partial y^{\prime 2}}
\end{gathered}
$$

subject to initial and boundary conditions

$$
\begin{gathered}
t^{\prime}>0: u^{\prime}=U_{0}, \frac{\partial T^{\prime}}{\partial y^{\prime}}=-\frac{h_{s}}{k} T^{\prime}, C^{\prime}=C_{w}^{\prime} \text { at } y^{\prime}=0 \\
u^{\prime}=0, T^{\prime}=T_{h}^{\prime}, C^{\prime}=C_{h}^{\prime} \text { at } y^{\prime}=h \\
t^{\prime} \leq 0: u^{\prime}=0, T^{\prime}=T_{h}^{\prime} C^{\prime}=C_{h}^{\prime} \text { for } 0 \leq y^{\prime} \leq h
\end{gathered}
$$

Here $u^{\prime}$ is the velocity of the fluid, $g$ is the acceleration due to gravity, $\beta$ is the volumetric coefficients of thermal expansion, while $\beta^{*}$ is the volumetric coefficients of concentration expansion, $t^{\prime}$ is time, $h$ is the distance between two vertical plates, $T^{\prime}$ is the temperature of the fluid, $T_{h}^{\prime}$ is the temperature of the plate at $y^{\prime}=h, C^{\prime}$ is the concentration of the fluid while $C_{h}^{\prime}$ is the concentration of the plate at $y^{\prime}=h, \rho$ is the density of the fluid, $C_{p}$ is specific heat at constant pressure, $D$ is mass diffusion coefficient, $q_{r}$ is the radiative heat flux in $y^{\prime}$-direction, $v$ is the kinematic viscosity, $k$ is the thermal conductivity of fluid, $C_{w}^{\prime}$ concentration of the fluid at $y^{\prime}=o$ and $h_{s}$ is the heat transfer coefficient. The nondimensional quantities are now introduced as:

$$
\begin{aligned}
y & =\frac{y^{\prime} U_{0}}{\nu}, t=\frac{t^{\prime} U_{0}^{2}}{\nu}, u=\frac{u^{\prime}}{U_{0}}, \\
\theta & =\frac{T^{\prime}-T_{h}^{\prime}}{T_{w}^{\prime}-T_{h}^{\prime}}, \mu=\rho \nu, H=\frac{U_{0} h}{\nu} \\
C & =\frac{C^{\prime}-C_{h}^{\prime}}{C_{w}^{\prime}-C_{h}^{\prime}},
\end{aligned}
$$

Equations (1)-(4) by using (5) will be reduced to the following non-dimensional form of equations:

$$
\begin{aligned}
& \frac{\partial u}{\partial t}=\frac{\partial^{2} u}{\partial y^{2}}+G r \theta+G m C \\
& 3 R \operatorname{Pr} \frac{\partial \theta}{\partial t}=(3 R+4) \frac{\partial^{2} \theta}{\partial y^{2}}
\end{aligned}
$$




$$
\frac{\partial C}{\partial t}=\frac{1}{S c} \frac{\partial^{2} C}{\partial y^{2}}
$$

and the initial and boundary conditions are

$t \leq o: u=o, \theta=o, C=o$ for $o \leq y \leq H$,

$t>0: u=1, \frac{\partial \theta}{\partial t}=-\gamma(\theta+1), C=1$ at $y=0$

$u=o, \theta=0, C=O$ at $y=H$

$G r=\frac{v g \beta\left(T_{w}^{\prime}-T_{h}^{\prime}\right)}{U_{0}^{3}} \quad$ is $\quad$ Grashof number $G m=\frac{v g \beta^{*}\left(C_{w}^{\prime}-C_{h}^{\prime}\right)}{U_{0}^{3}} \quad$ is mass Grashof number $R=\frac{k k^{*}}{4 \sigma^{*} T_{h}^{3}} \quad$ is radiation parameter, $\operatorname{Pr}=\frac{\mu C_{p}}{k} \quad$ in Prandtl number and $\gamma=\frac{h_{s}}{k}$ is newtonian heating parameter. The solutions of velocity and temperature after solving equations (6), (7) and (8) under the initial and boundary conditions ( 9 ) by using Laplace transform technique are obtained as

$$
\begin{gathered}
u(y, t)=\sum_{n=0}^{\infty}\left[f_{1}(a, t)-f_{1}(b, t\right. \\
+a_{1} \sum_{n=0}^{\infty} \sum_{m=0}^{n}(-1)^{m}\left[f_{3}(c, t)-f_{3}(d, t)\right. \\
\left.-f_{3}(g, t)+f_{3}(h, t)\right]+a_{2} \sum_{n=0}^{\infty}\left[-f_{4}(a, t)\right. \\
\left.+f_{4}(b, t)+f_{4}(a \sqrt{S c}, t)-f_{4}(b \sqrt{S c}, t)\right] \\
a_{1} \sum_{n=0}^{\infty}\left[-f_{3}(a \sqrt{A}, t)+f_{3}(b \sqrt{A}, t)\right] \\
\theta(y, t)=\sum_{n=0}^{\infty}(-1)^{n} k_{1}\left[f_{2}(a \sqrt{A}, t)-\right. \\
\left.f_{2}(b \sqrt{A}, t)\right]+k_{2}\left[f_{2}(a \sqrt{A}, t)-\right. \\
\left.f_{2}(b \sqrt{A}, t)\right]
\end{gathered}
$$

$$
C(y, t)=\sum_{n=0}^{\infty}\left[f_{1}(a \sqrt{S c}, t)-f_{1}(b \sqrt{S c}, t)\right]
$$

where

$$
a=2 n H+y, \quad b=2 n H+2 H-y,
$$

$$
c=2 m H \sqrt{A}+a, \quad d=2 m H \sqrt{A}+b,
$$

$$
g=2 m H \sqrt{A}+2 H \sqrt{A}+a
$$

$$
h=2 m H \sqrt{A}+2 H \sqrt{A}+b
$$

$$
\begin{gathered}
k_{1}=\frac{\gamma}{\sqrt{A}}, \quad k_{2}=\frac{\gamma^{2}}{\sqrt{A}}, \quad A=\frac{3 R P r}{3 R+4} \\
a_{1}=\frac{G r\left(\gamma+\gamma^{2} H\right)}{(A-1) \sqrt{A}}, \quad a_{2}=\frac{G m}{S c-1}
\end{gathered}
$$

$$
f_{1}(z, t)=\operatorname{Erfc}\left(\frac{z}{2 \sqrt{t}}\right)
$$

$$
f_{2}(z, t)=2 \sqrt{\frac{t}{\pi}} e^{\frac{-z^{2}}{4 t}}-z \operatorname{Erfc}\left(\frac{z}{2 \sqrt{t}}\right)
$$

$$
\begin{array}{r}
f_{3}(z, t)=\frac{1}{3}\left(4 t+z^{2}\right) \sqrt{\frac{t}{\pi}} e^{\frac{-z^{2}}{4 t}}-\left(z t+\frac{z^{3}}{6}\right) \\
\operatorname{Erfc}\left(\frac{z}{2 \sqrt{t}}\right) \\
f_{4}(z, t)=\left(\frac{z^{2}}{2}+t\right) \operatorname{Erfc}\left(\frac{z}{2 \sqrt{t}}\right)-z \sqrt{\frac{t}{\pi}} e^{\frac{-z^{2}}{4 t}}
\end{array}
$$


Here, $z$ is a dummy variable, $f_{1}, f_{2}, f_{3}, f_{4}$ are functions of dummy variable and $\operatorname{Erfc}(\mathrm{z})=1-\frac{2}{\sqrt{\pi}} \int_{0}^{\pi} e^{-t^{2}} d t$ is is the complementary error function. Using solutions (10) and (11), the skin friction and the Nusselt number are given by,

$$
\tau=\frac{\tau^{\prime} h}{\mu U}=-\left.\frac{\partial u}{\partial y}\right|_{y=0}
$$

and

$$
N u=\left.\frac{q h}{k\left(T^{\prime}-T^{\prime} h\right)} \frac{\partial T^{\prime}}{\partial y^{\prime}}\right|_{y=0}=1+\frac{1}{\theta(0, t)}
$$

Skin friction and Nusselt number are computed using Mathematic software.

\section{RESULTS AND DISCUSSIONS}

In order to get the physical insight of the problem, the graphical results of the temperature field, velocity field, concentration field, skin friction and Nusselt number are plotted for different parameters such as Newtonian heating parameter, Schmidt number, Prandtl number and radiation parameter.

Figure 2 shows that increasing $S c$ number will decrease the concentration of the fluid flow. $S c$ number physically relates the relative thickness of the hydrodynamic layer and mass transfer boundary layer, when $S c$ number increases will cause the thickness increases and causing concentration of the fluid flow decreases.

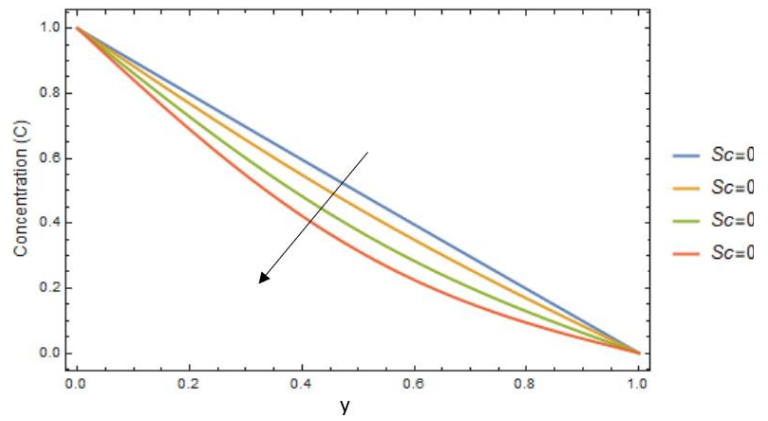

Figure 2. Concentration profiles with different $S c$
While Figures 3-5 show temperature profiles with different parameters. Figure 3 describe the effect of Newtonian heating parameter $\gamma$ on the temperature profile. Increasing $\gamma$ will increase the temperature. It is because $\gamma$ acts as a boosting agent which enhance the temperature of the flow. While Figure 4 shows the effect of thermal radiation $R$ on the temperature profile. Increasing $R$ results in decreasing the temperature. Figure 5 shows the effect of Prandtl number $P r$ on the temperature profile. Physically, profile in this figure occurred is due to the fact that increasing $\mathrm{Pr}$ number, the thermal conductivity of fluid decreases and viscosity of the fluids increases and as a result the thermal boundary layer decreases with increasing $P r$ number.

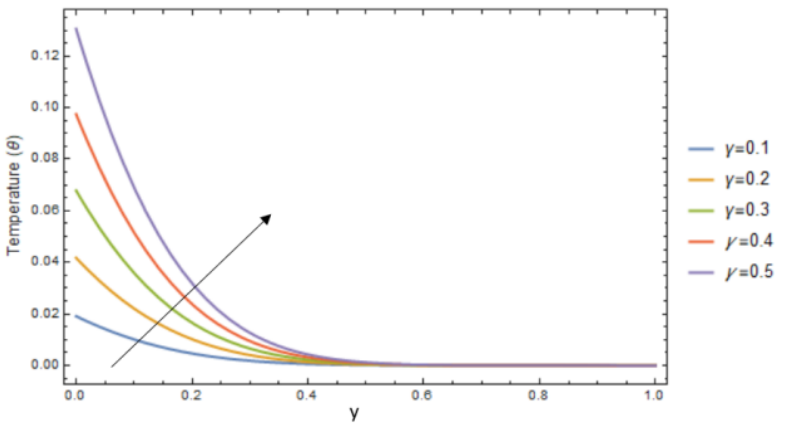

Figure3. Temperature files with different $\gamma$ pro

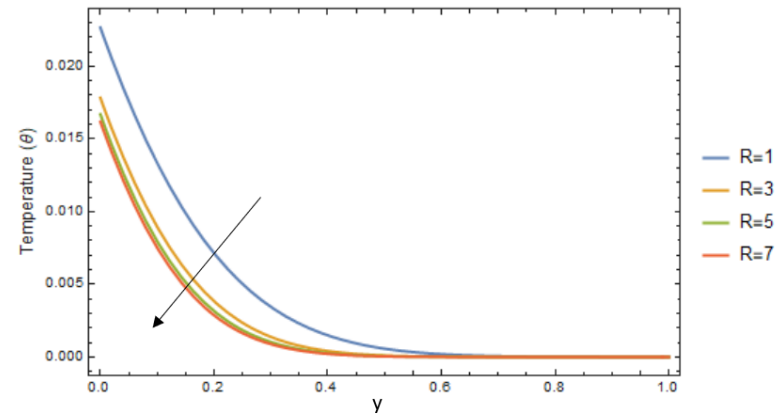

Figure 4. Temperature profiles with different $\mathrm{R}$

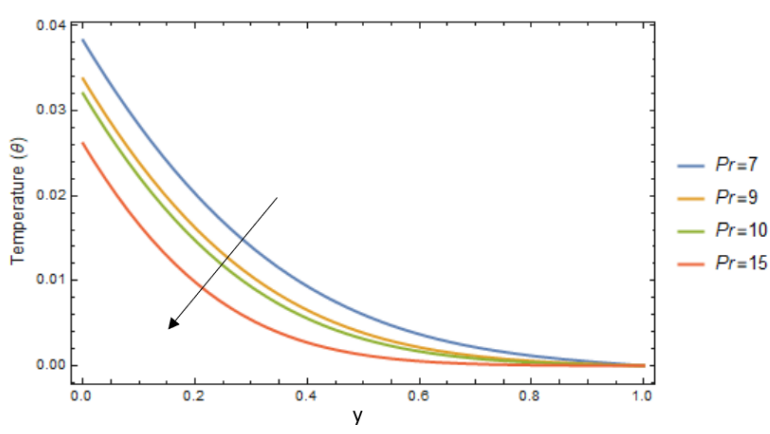

Figure 5. Temperature profiles with different $\operatorname{Pr}$ 
Figures 6-9 show the effects of different parameters on the velocity profile. From Figure 6 it is observed that increasing the values of $\gamma$ increases the velocity. It is found that as the Newtonian heating parameter increases, the density of the fluid decreases and the momentum boundary layer thickness increases and as a result, the velocity increases within the boundary layer. Figure 7 shows that increasing $\mathrm{R}$ enhances convective flow in such way that the flow of velocity increases. While Figure 8 shows that increasing $\operatorname{Pr}$ number cause increases in velocity. The reason is that smaller Pris equivalent to the increases in thermal conductivity if the fluid, and heat is able to diffuse away from the heated surface more rapidly for higher values of $\mathrm{Pr}$. In Figure 9 shows that increasing $S c$ number increases the velocity since higher $S c$ number will cause the concentration decreases so the velocity will be increases.

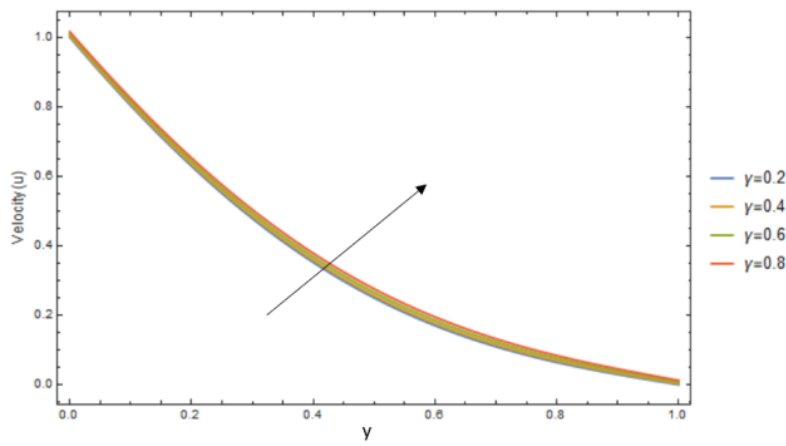

Figure 6. Velocity profiles with different $\gamma$

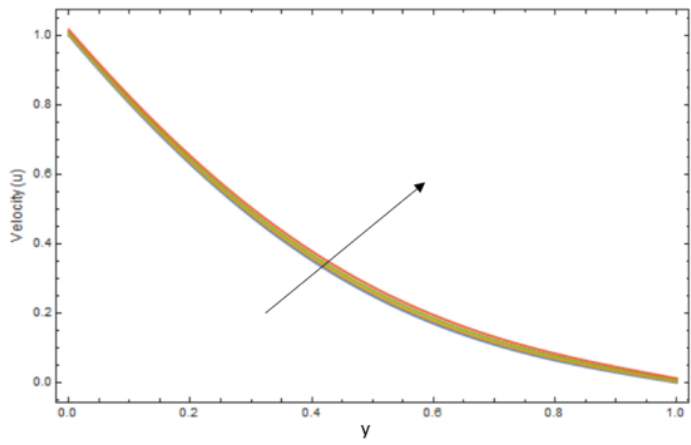

Figure 7. Velocity profiles with different $\mathrm{R}$

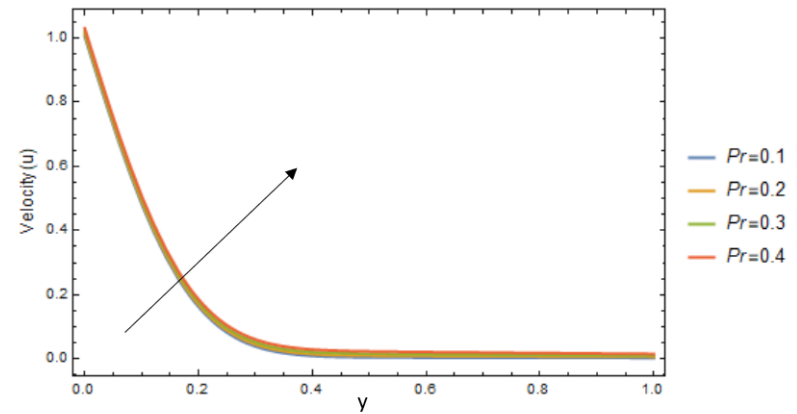

Figure 8. Velocity profiles with different Pr

In Figures 10 - 13 illustrates the variation of skin friction and Nusselt number against time, $t$ with different effects of thermal radiation parameter and Newtonian heating parameter, Figures 10 and 12 show Nusselt number and skin friction with different $\gamma$ parameter. The effect of increasing $\gamma$ parameter cause the Nusselt number to increase but decreases the skin friction. While in Figure 11 and 13 show effect of $R$ towards Nusselt number and skin friction. Increasing $R$ decreases the Nusselt number and skin friction.

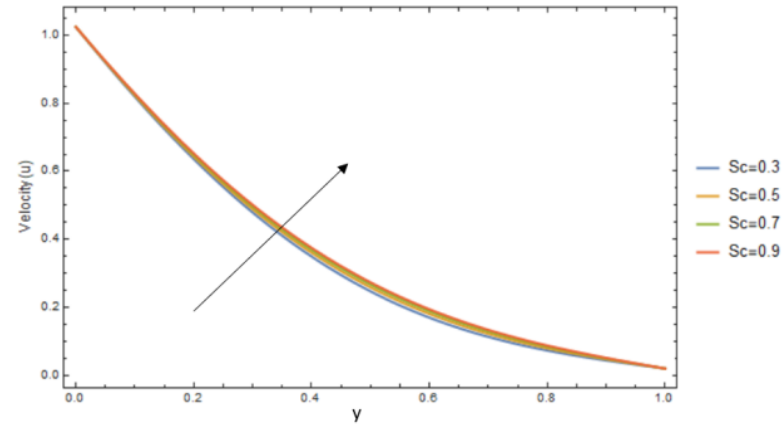

Figure 9. Velocity profiles with different $S c$

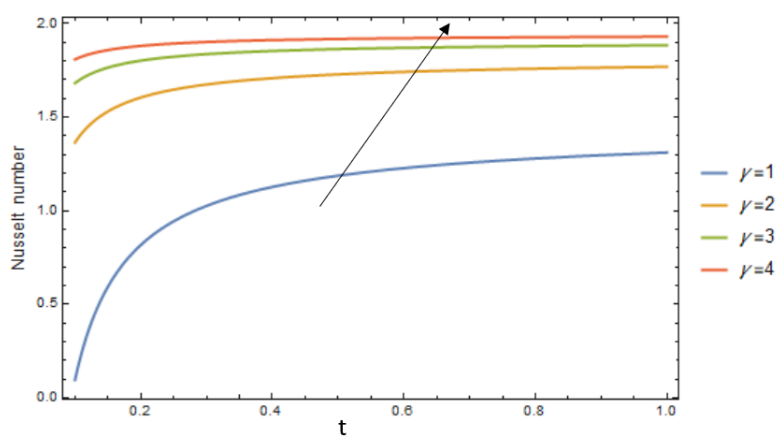

Figure 10. Nusselt number graph with different $\gamma$ 


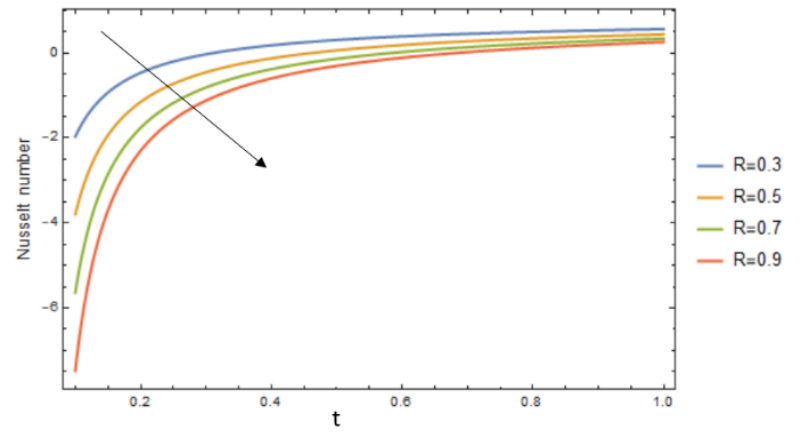

Figure 11. Nusselt number graph with different $R$

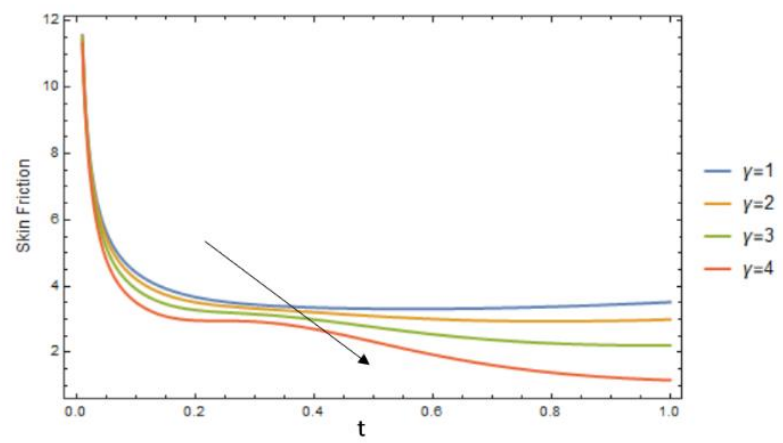

Figure 12. Skin friction with different $\gamma$

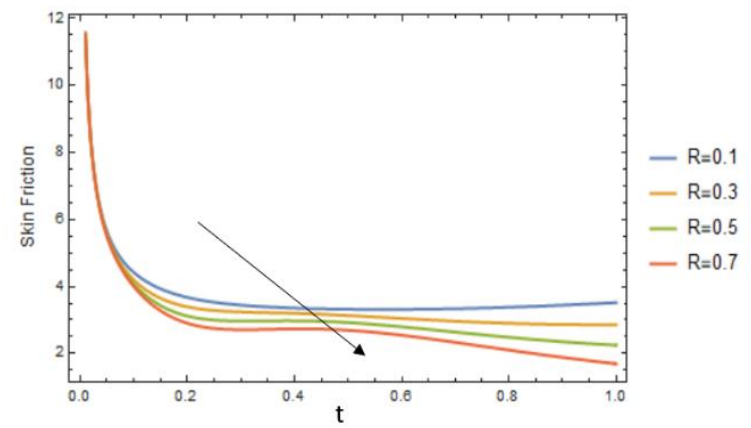

Figure 13. Skin friction with different $R$

\section{CONCLUSION}

Free convection boundary layer flow with radiation between two vertical parallel plates with Newtonian heating and mass diffusion have been studied. The dimensionless governing linear partial differential equations are solved using Laplace Transform method. From the graphical results velocity increases but temperature decreases when the $\operatorname{Pr}$ number increases. The results show that increasing $S c$ number will decrease the concentration. Furthermore, it is observed that the velocity increases with increase in either thermal radiation or Newtonian heating parameter. The temperature profile increases with the increase in the Newtonian heating parameter but decreases for thermal radiation parameter. The results obtained show that the effect of Newtonian heating parameter increases the Nusselt number but decreases the skin friction. Nusselt number decreases when radiation increases while skin friction decreases when Radiation parameter increases. The flow characteristics observed in this paper are found similar with Das et al. (2012) that investigated vertical plate with Newtonian heating and radiation. Also, it is interesting to mention that exact solutions of velocity (10), temperature (11) and concentration (12) are fully satisfied the non-dimensional equations (6) to (8). These ensure that all the obtained solutions are correct. The exact solutions obtained in this study are significant not only because they are solutions of some fundamental flows, but they serve as accuracy standards for approximate method. 


\section{ACKNOWLEDGEMENT}

The authors would like to express their appreciation for the support of the sponsors from UTM Zamalah and acknowledge Ministry of Education (MOE) and Research Management Centre-UTM, Universiti Teknologi Malaysia (UTM) for the financial support through vote number $5 \mathrm{FoO} 4$ for this research.

\section{REFERENCES}

Das, S., Mandal, C. and Jana, R. 2012, Radiation effects on unsteady free convection flow past a vertical with Newtonian heating. International Journal of Computer Applications, 41(13).

Hamza, M. 2016, Free convection slip flow of an exothermic fluid in a convectively heated vertical channel. Ain Shams Engineering Journal.

Jha, B. K. and Ajibade, A. O. 2011, Diffusion-thermo effects on free convective heat and mass transfer flow in a vertical channel with symmetric boundary conditions. Journal of Heat Transfer, 133(5), 052502.

Lesnic, D., Ingham, D. B., Pop, I. and Storr, C. 2004, Free convection boundary-layer flow above a nearly horizontal surface in a porous medium with newtonian heating,40,665-672.

Marneni, N. 2008, Transient free convection flow between two long vertical parallel plates with constant temperature and mass diffusion. In Proceedings of the World Congress on Engineering (WCE), London, UK, (11).

Narahari, M. 2012, Transient free convection flow between long vertical parallel plates with ramped wall temperature at one boundary in the presence of thermal radiation and constant mass diffusion. Meccanica, 47(8), 1961-1976.

Paul, T., Jha, B. K. and Singh, A. K. 1996, Transient free convective flow in a vertical channel with constant temperature and constant heat flux on walls, 32, o-2.
Rajput, U and Sahu, P. 2011, Combined effects of chemical reactions and heat generation/absorption on unsteady transient free convection MHD flow between two long vertical parallel plates through a porous medium with constant temperature and mass diffusion. Elixir Appl. Math, 39, 4855-4859.

Rajput, U. and Sahu, P. 2011, Transient free convection MHD flow between two long vertical parallel plates with constant temperature and variable mass diffusion. Journal of Math. Analysis, 34(5), 1665-1671.

Singh, A., Gholami, H. R. and Soundalgekar, V. (1996) Transient free convection flow between two vertical parallel plates. Heat and mass transfer, 31(5), 329331.

Shehzad, S., Hayat, T., Alhuthali, M. and Asghar, S. 2014, MHD three-dimensional flow of Jeffrey fluid with Newtonian heating. Journal of Central South University, 21(4), 1428-1433. 